\title{
BMJ Open Women's well-being and functioning after evidence-based antenatal care: a protocol for a systematic review of intervention studies
}

\author{
Carla Betina Andreucci (D , , ${ }^{1}$ Veronique Filippi, ${ }^{2}$ Jose Guilherme Cecatti (D) ${ }^{3}$
}

To cite: Andreucci CB, Filippi V, Cecatti JG. Women's well-being and functioning after evidencebased antenatal care: a protocol for a systematic review of intervention studies. BMJ Open 2021;11:e042667. doi:10.1136/ bmjopen-2020-042667

- Prepublication history for this paper is available online. To view these files, please visit the journal online (http://dx.doi. org/10.1136/bmjopen-2020042667).

Received 11 July 2020

Revised 12 December 2020

Accepted 25 January 2021

Check for updates

(C) Author(s) (or their employer(s)) 2021. Re-use permitted under CC BY-NC. No commercial re-use. See rights and permissions. Published by BMJ.

${ }^{1}$ Department of Medicine, Federal University of Sao Carlos, Sao Carlos, Brazil

${ }^{2}$ Department of Infectious Disease Epidemiology, London School of Hygiene and Tropical Medicine, London, UK

${ }^{3}$ School of Medical Sciences, University of Campinas, Campinas, Brazil

Correspondence to Dr Carla Betina Andreucci; carlapolido@ufscar.br

\section{ABSTRACT}

Introduction The 2016 WHO antenatal guidelines propose evidence-based recommendations to improve maternal outcomes. We aim to complement these recommendations by describing and estimating the effects of the interventions recommended by WHO on maternal well-being or functioning.

Methods and analysis We will conduct a systematic review of experimental and quasi-experimental studies evaluating women's well-being or functioning following the implementation of evidence-based antenatal interventions, published in peer-reviewed journals through a 15-year interval (2005-2020). The lead reviewer will screen all records identified at MEDLINE, EMBASE, CINAHL Plus, LILACS and SCiELO. Two other reviewers will control screening strategy quality. Quality and risk of bias will be assessed using a specially designed instrument. Data synthesis will consider the instruments applied, how often they were used, conditions/interventions for positive or negative effects documented, statistical measures used to document effectiveness and how results were presented. A random-effects meta-analysis comparing frequently used instruments may be conducted.

Ethics and dissemination The study will be a systematic review with no human beings' involvement, therefore not requiring ethical approval. Findings will be disseminated through peer-reviewed publication and scientific events. PROSPERO registration number CRD42019143436.

\section{INTRODUCTION}

Antenatal care (ANC) is a key intervention to prevent women's morbidity and mortality. Maternal morbidity has been recently defined by the WHO Maternal Morbidity Working Group (MMWG) as 'any health condition attributed to and/or complicating pregnancy and childbirth that has a negative impact on the woman's well-being and/or functioning'. Well-being refers to women's health-related quality of life (HRQoL), including their satisfaction with their health status. ${ }^{2}$ Functioning relates to both physical and cognitive organic functions, as well as activities and participation in the family or in society, and it is the positive correlate of disability. ${ }^{3}{ }^{4}$ Providing

\section{Strengths and limitations of this study}

- Our systematic review will address an information gap on the extent to which key antenatal care interventions may have positive or negative effects on women's well-being and functioning.

- This will be the first systematic review on effects of antenatal interventions on maternal well-being and functioning.

- Our results could be directly integrated in future antenatal guidelines or recommendations, and they could also lead research teams working on future guidelines to include their own analysis of the effectiveness of interventions using functioning and well-being as outcomes.

- One study limitation will be the study being solely based on interventions recommended in the 2016 WHO antenatal care guidelines, with only five interventions meeting our criteria of moderate/high certainty evidence of effectiveness.

well-established healthcare for women during pregnancy is known to be an effective measure to enhance obstetric outcomes. Therefore, evidence-based recommendations that might reduce maternal morbidity and mortality have been brought together in many global and country-specific guidelines on ANC. .7 $^{5-7}$

In 2016, the WHO published its antenatal guidelines summarising and updating recommendations available to improve maternal and perinatal health. The document added an innovative human rights-based approach for management of pregnancy-related complications, prioritising a person-centred model that results in a positive pregnancy experience with ANC. The 2016 WHO antenatal guidelines are a detailed manual that presents a narrative description of several recommendations for specific health outcomes, and includes the level of evidence of the proposed interventions, classified as high, moderate and low certainty of evidence. ${ }^{8}$ Nonetheless, to date no guidelines have-so far as 
we are aware-addressed how recommended interventions influence women's well-being and/or functioning (both of which are key elements of the broader maternal morbidity concept).

Many validated instruments exist that either measure well-being or functioning through several predetermined dimensions, concerned for example with physical and psychological health status and functions, abilities to perform daily activities, social participation, self-esteem and satisfaction with heath. ${ }^{9}$ Frequently used instruments of HRQoL include 36-Item Short Form Survey and WHO-Quality of Life, while WHO-Disability Assessment Schedule is a commonly used functioning instrument. ${ }^{10-12}$ However, there is no consensus about which tool(s) most satisfactorily evaluate well-being and functioning during pregnancy and the postpartum period. Available instruments may include interviewer-administered, selfadministered and proxy-administered questionnaires, using scores to identify women with important needs, ${ }^{13-16}$ as well as qualitative evaluations. ${ }^{10-1217-20}$ Several maternal health or disease-specific questionnaires also exist for assessing HRQoL during pregnancy. ${ }^{21-23}$

Maternal health has been under the scope of the Sustainable Development Goals (SDG) to be achieved until 2030, especially through the highlighted 'good health and well-being' (SDG 3) and 'gender equality' (SDG 5) ${ }^{24}$ goals. Thus, together with reduction of morbidity and mortality, future antenatal measures/actions/recommendations should include promoting women's well-being and functioning, comprising a positive pregnancy experience and preventing violence. It is crucial to gather information available on which key healthcare interventions may have a positive effect on women's well-being and/or functioning, so that they might be tested in intervention studies among different populations and settings in the future. From a long-term perspective, such information could be integrated in forthcoming ANC guidelines or recommendations.

Our objectives will be to compare, appraise and summarise studies focusing on evidence-based interventions that are recommended in the WHO ANC guidelines and were applied during ANC, and that have/have not influenced maternal well-being or functioning. In the WHO ANC guidelines, evidence-based interventions consisted of treatment, procedures or more complex programmes that were evaluated using experimental and quasi-experimental studies as well as observational studies.

\section{METHODS AND ANALYSIS}

This will be a systematic review of experimental and quasiexperimental studies evaluating women's well-being and functioning associated with or as a result of the implementation of selected evidence-based antenatal interventions. We aim to analyse whether well-established interventions applied to pregnant women have affected their well-being and functioning.
The period covered will be studies published from 2005 until 2020. The initial search was already performed, and selection of studies is currently under quality analysis. There will be a language restriction to articles published in English, French, Portuguese and Spanish. The systematic review will follow the PRISMA-P statement (Preferred Reporting Items for Systematic Review and Meta-analysis Protocols) ${ }^{25}$ and has been registered at the International Prospective Register of Systematic Reviews.

For the present review, exposure will be interventions recommended for ANC because they reduce the occurrence of direct or indirect obstetric conditions, and outcome will be women's well-being and/or functioning during pregnancy or after childbirth after receiving the recommended ANC interventions.

\section{Eligibility criteria}

There are no country/region inclusion or exclusion criteria. Included studies will have been conducted in primary care facilities, during home-based care or hospital-based care. The present review will include studies whose subjects are pregnant women at any gestational age, who have received one of the preselected interventions (outlined in the next paragraph), and who were evaluated from a well-being and functioning perspective (specifically designed tools/instruments or qualitative assessment) at any point in time during or after pregnancy. The inclusion of the postnatal period may provide data on longer term outcomes concerning well-being and functioning related to pregnancy.

The selection of interventions was based entirely on the 2016 WHO Antenatal Guideline. ${ }^{8}$ The WHO guidelines were intended to promote obstetric care within a broader framework, beyond prevention of death and morbidity, and to prioritise the applicability of interventions in low-income and middle-income countries. We selected the WHO guidelines on account of their wide international circulation, as well as their use as a model for health policies around the world. Health promotion and identification of risk factors are within the scope of these guidelines, whereas treatment of complications and concurrent diseases are not. In order to account for women's perspectives about the care provided to them, systematic reviews on women's expectations on ANC were carried out, and the final version of the document was intended to promote well-being as part of the "positive pregnancy experience'.

The WHO guidelines list 49 recommendations grouped into 5 'types of interventions' and concerning 10 'maternal outcomes of interest' (figure 1), as well as fetal and neonatal outcomes. Among the 49 recommendations, 10 were included into the 2016 document from existing WHO guidelines approved by the review committee for the 2016 document. Evidence on the effectiveness of interventions was obtained from 47 systematic reviews. Additionally, findings from a scoping review on women's understanding of a positive ANC experience within high-resource, middle-resource and low-resource 


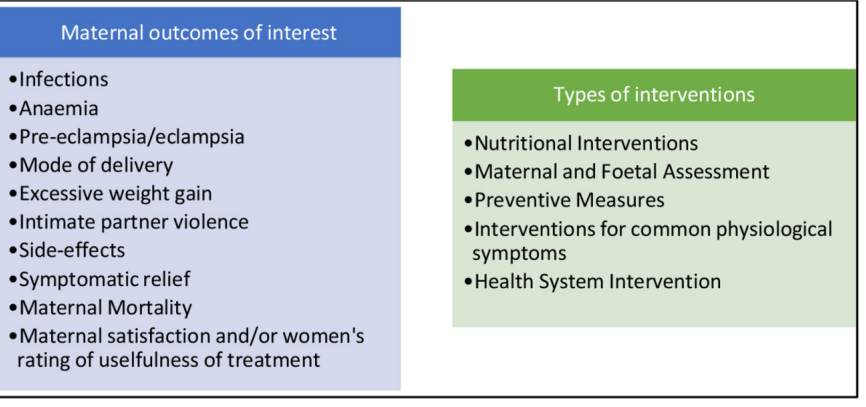

Figure 1 Maternal Outcomes of interest and types of interventions according to 2016 WHO Antenatal Guideline.

settings suggested the importance of effective clinical practices, relevant and timely information, and psychosocial and emotional support. ${ }^{26}$ Consideration of women's perspectives was therefore applied together with quantitative evidence to evaluate the recommendations. The GRADE (Grading of Recommendations, Assessment, Development and Evaluations) approach was applied to appraise the quality of quantitative evidence. ${ }^{27}$ The quality of evidence was rated as 'high', 'moderate', 'low' or 'very low', with randomised controlled trials (RCTs) providing 'high-quality' evidence, while non-randomised trials and observational studies provided 'low-quality or moderate-quality' evidence. Qualitative reviews were appraised using the GRADE-CERQual tool. ${ }^{28}$

For the present systematic review, we reviewed the 49 recommendations findings with respect to their effects on 'maternal outcomes of interest' only, in order to ascertain the certainty of the evidence for the effectiveness of the intervention on women's health improvement. We did not include maternal well-being and functioning outcomes for selecting interventions at this point. We found five interventions that were presented as having either a high or a moderate level of certainty of evidence associated with one or more of the listed 'maternal outcomes of interest'. Those five interventions with high and moderate level of certainty of evidence will be further assessed in our systematic review to establish their effectiveness in improving maternal well-being or functioning.

The five interventions described as improving maternal outcomes that will therefore be included in our systematic review are listed below:

1. Daily iron and folic acid supplementation: reduces puerperal infections and anaemia (when daily and intermittent supplementation were compared, favouring daily).

2. Multiple micronutrient supplementation: reduces anaemia (similar effect to iron and folic acid supplementation).

3. Vitamin A supplementation: reduces anaemia (and night blindness at affected populations).

4. Diet and/or exercise: prevents hypertension and excessive weight gain (but not pre-eclampsia/eclampsia).

5. Midwife continuity of care: increases vaginal birth rates.
Data management

We will search MEDLINE, EMBASE, CINAHL Plus, LILACS and SciELO with customised search strategies for each electronic database according to their individual subject headings, syntax and searching structure. The search will combine free text and Medical Subject Headings terms for the proposed interventions and maternal outcomes. For the maternal outcomes, we will apply keywords and index terms for the concepts of HRQoL, well-being and functioning. The search strategy is shown in online supplemental appendix 1.

Initially, CBA (lead reviewer) will select manuscripts by scrutinising their titles and abstracts, excluding those that are not related to the proposed objectives. To maximise the identification of all relevant results and ensure quality control, VF and JGC will look at a proportion of manuscripts to check whether they were included or not, until perfect matching in selection has been obtained. Following this step, we will assess the full text of the remaining documents, discarding irrelevant records, unless they can provide additional interpretation data. CBA will extract the data from relevant studies using a screening form specifically designed for the present review (online supplemental appendix 2) and examining their reference lists to identify additional papers for inclusion. She will also scrutinise the reference lists of relevant literature reviews identified by our search strategy.

Prior to the final analysis, we will conduct a rerun of the searching strategy in order to check for more recent studies not initially included. Search results will be managed in EndNote and extracted data will be entered in an Excel spreadsheet.

We will list all instruments/questionnaires used for well-being and/or functioning measurement, and studies that applied the same instrument will be compared.

We will prepare a PRISMA flowchart to display results of the search, screening, review and extraction in a sequential manner, and a list of rejected articles along with the reasons for their exclusion.

\section{Assessment of study quality/risk of bias}

Two reviewers will appraise the data, and a third reviewer will be available for discussion if they are not in consensus. The planned analysis is a meta-analysis of published data in selected studies. The quality and risk of bias will be assessed using a tool specifically designed for this purpose, based on version 2 of Cochrane's tool for assessing bias in randomised trials, ${ }^{29}$ and, if necessary, using statistical approach. For this, we included the bias domains and risk-of-bias questions in the study screening form (online supplemental appendix 2).

The dimensions analysed for bias include:

1. Sampling: What is the intended study population? (pregnant women with or without a condition; all pregnant women with a condition in the community; pregnant women attending health services; pregnant women with a condition attending health services; special categories of women). 
- Detailed information written in full (extracted from manuscript) for the following:

- Are the respondents representative of the intended study population?

- Is there an adequate description of data collection location, including reasons for choice?

- Is there an adequate description of inclusion and exclusion criteria? (sociodemographic characteristics of the study population such as age, gestational length, ethnicity, level of education, parity, previous health condition and so on).

- Ethical issues (is there a consent form?).

2. Completeness: Are there enough women interviewed and followed up?

- Detailed information written in full (extracted from manuscript) and then simple yes/no answers for the following:

- Is the sample size calculation adequate?

- What was the follow-up rate? Are particular subgroups of women more likely to be lost to followup? Are reasons for loss of follow-up explained? (explanations in full text).

3. Study design: Is the study design described properly?

- Detailed information written in full (extracted from manuscript) and then simple yes/no answers for the following:

- Is the methodology adequate for drawing cited results/conclusions?

- If the study is an RCT, was randomisation properly conducted?

- If the study is an RCT, are investigators ascertaining outcomes blind to intervention allocation?

- What certainty is there that health-related functioning/well-being is a consequence of the evidence-based antenatal or postnatal intervention?

4. Comparison: Is there a comparison group of pregnant/postpartum women without evidence-based intervention?

- Detailed information for each group written in full (extracted from manuscript) and then simple yes/ no answers for the following:

- If the study has a quasi-experimental design, are there concurrent comparison groups and/or 'before and after' evaluations?

- What was the content of care in the control group?

5. Validation: If a standardised tool was used, was the validity of the tool known or established for the population of interest (pregnant or postpartum women; women of reproductive age; preferably in the same geographical location)? (yes/no).

Do applied well-being/functioning instruments have appropriate psychometric properties? (yes/no).

6. Intervention: Did the authors describe the content and implementation of the selected intervention in sufficient detail? (yes/no).
7. Conceptualisation: Did the authors provide a definition of health-related functioning/well-being? (yes/ no).

Did the authors describe well-being and functioning instruments used? Are they correctly applied in the study? (yes/no).

\section{Data synthesis}

Among selected studies with any maternal outcome, our synthesis will consider the following:

- What were the instruments applied to evaluate wellbeing/functioning and how often were they used? What type of measures do they use?

- For which interventions have positive or negative effects been documented?

- What were the measures of effects used to quantify well-being or functioning?

- How were the results presented regarding impact on well-being/functioning?

- Can results from different studies be combined? (same instrument/methodology).

The findings of the systematic review will be displayed in tables with studies' characteristics (sample, design, results). A table with listed studies and instruments will be provided, detailing methods, participants and results, as well as the appraisal of the quality of selected studies and questionnaires. We will apply GRADE to summarise the level of evidence available from our systematic review findings. ${ }^{27}$ Different HRQoL, well-being and functioning tools will be described according to their scoring systems, and then categorised in order to display their effects in an organised panel. We will calculate the proportion of studies with positive or negative effects for each selected intervention, and present the range of effects in a table format.

Finally, a narrative of the range of impact on wellbeing/functioning will be developed to summarise the findings. Fixed-effects or random-effects meta-analyses may be considered to analyse the results of the most frequently used well-being and functioning instruments, depending on the homogeneity of exposure/outcome characteristics.

\section{Patient and public involvement}

Patients or the public were not involved in the design, or conduct, or reporting, or dissemination plans of our study.

\section{DISCUSSION}

Recently, a standardised tool (WOICE) was developed by the WHO MMWG to identify maternal morbidity, recognising that there are many aspects of women's lives which may be affected by ill-health during pregnancy or delivery. ${ }^{12}$ Applications of the WOICE tool revealed that the prevalence of self-reported ill-health was higher than appraised by health professionals, ${ }^{30}$ a conclusion found in other studies which compared self-reported 
data with diagnoses. ${ }^{31} 32$ These findings suggest that pregnant women's own perceptions of their health allow for a more comprehensive identification of the health-related burden of pregnancy and complications than diagnoses alone.

We plan to identify and describe good quality studies evaluating the influence of well-established antenatal interventions/recommendations on maternal well-being and functioning. Furthermore, we plan to meta-analyse findings from studies that applied the same measurement instrument and provide a summarised narrative of findings from studies that could not be directly compared because of a lack of common instrument, but provided answers to our research question.

By the same token, our systematic review will provide much needed comprehensive insights on whether selected evidence-based interventions recommended for use during ANC have an impact on women's self-reported well-being and/or functioning during pregnancy or postnatally, as well as on diagnoses of clinical conditions. In addition, the review is likely to provide useful methodological insights on the instruments applied for this assessment, since it has been suggested that no currently available instrument is sufficiently comprehensive to capture the particularities of functioning and wellbeing during the pregnancy, childbirth and postpartum period, and that there is a need for the development of a new maternity-specific tool. ${ }^{43}$ We believe our systematic review is likely to highlight interventions for which further evidence-based investigation is required, since documenting well-being and functioning during pregnancy and childbirth is relatively new, particularly in lowincome and middle-income settings.

Pregnancy, childbirth and the postpartum period constitute a unique period during which women need to cope with physiological modifications while taking care of their child and frequently their household and professional career. The broader approach towards women's health beyond reproductive aspects was already pointed as key measure to achieve sustainable development. ${ }^{34}$ The strain of being pregnant, giving birth and facing the postpartum period may negatively impact women's well-being and functioning, even more if during these circumstances women also struggle with morbidity or hypothetical unwanted outcomes from antenatal interventions. ${ }^{15} 3536$ Currently, we have no satisfactory means to properly identify and respond to these difficulties and empower women to thrive. Our proposed systematic review aligns with the objectives of the SDG in promoting well-being under a gender perspective. ${ }^{37}$

We searched through several antenatal guidelines including, for example, The National Institute for Health and Care Excellence, the American College of Obstetrics and Gynaecology, the International Federation of Gynaecology and Obstetrics, the Society of Obstetricians and Gynaecologists of Canada, the Pregnancy Care Guidelines from Australian Department of Health, guidelines from the Collège National des Gynécologues et Obstétriciens
Français, guidelines for maternity care in South Africa and Brazilian guidelines for routine ANC. To the best of our knowledge, no available guidelines include an assessment of women's well-being or functioning as part of their evaluation of the evidence base for their recommended interventions. ${ }^{5638-43}$ Future clinical guidelines should not only recommend clinical evidence-based measures based on the treatment of diseases, but also take into account the possible positive or negative impact of interventions on women's HRQoL or functioning. ${ }^{114}$ Appraising available evidence-based interventions recommended in the WHO ANC guidelines that have influenced maternal well-being or functioning might provide information to be integrated in forthcoming ANC guidelines or recommendations, under a women's well-being perspective.

\section{ETHICS AND DISSEMINATION}

The study will be a systematic review based on secondary data, therefore not requiring ethical approval. Patients were not directly involved in the study design, therefore dissemination of findings to participants is not applicable.

Any further amendments to this protocol will be documented and recorded in bibliographical databases.

The results will be disseminated using green or gold open access to ensure universal access for researchers, academics, caregivers and policymakers.

Twitter Veronique Filippi @1verofilippi

Acknowledgements The authors thank the Center for Studies on Reproductive Health of Campinas (CEMICAMP) for supporting the publication of this manuscript. The authors also thank the Maternal and Neonatal Health Group from the Faculty of Epidemiology and Public Health at The London School of Hygiene and Tropical Medicine (LSHTM) for the important inputs in developing the study during CBA's postdoctoral project at the institution. We thank Clara Calvert from LSHTM for reviewing an earlier version of this paper.

Contributors CBA and VF conceptualised and designed the protocol. CBA, VF and JGC defined the concepts and search items, data extraction process as well as methodological appraisal of the studies. All authors have approved and contributed to the final written manuscript.

Funding The authors have not declared a specific grant for this research from any funding agency in the public, commercial or not-for-profit sectors.

Competing interests None declared.

Patient consent for publication Not required.

Provenance and peer review Not commissioned; externally peer reviewed.

Supplemental material This content has been supplied by the author(s). It has not been vetted by BMJ Publishing Group Limited (BMJ) and may not have been peer-reviewed. Any opinions or recommendations discussed are solely those of the author(s) and are not endorsed by BMJ. BMJ disclaims all liability and responsibility arising from any reliance placed on the content. Where the content includes any translated material, BMJ does not warrant the accuracy and reliability of the translations (including but not limited to local regulations, clinical guidelines, terminology, drug names and drug dosages), and is not responsible for any error and/or omissions arising from translation and adaptation or otherwise.

Open access This is an open access article distributed in accordance with the Creative Commons Attribution Non Commercial (CC BY-NC 4.0) license, which permits others to distribute, remix, adapt, build upon this work non-commercially, and license their derivative works on different terms, provided the original work is properly cited, appropriate credit is given, any changes made indicated, and the use is non-commercial. See: http://creativecommons.org/licenses/by-nc/4.0/. 


\section{ORCID iDs}

Carla Betina Andreucci http://orcid.org/0000-0002-5590-108X Jose Guilherme Cecatti http://orcid.org/0000-0003-1285-8445

\section{REFERENCES}

1 Chou D, Tunçalp Özge, Firoz T, et al. Constructing maternal morbidity - towards a standard tool to measure and monitor maternal health beyond mortality. BMC Pregnancy Childbirth 2016;16:45.

2 Filippi V, Chou D, Barreix M, et al. (MMWG) the WHOMMWG. A new conceptual framework for maternal morbidity. Int J Gynecol Obstet [Internet] 2018;141:4-9.

3 WHO. Towards a common language for functioning, disability and health: ICF. Int Classif [Internet], 2002. Available: http://www.who.int/ classifications/icf/training/icfbeginnersguide.pdf

4 Machiyama K, Hirose A, Cresswell JA, et al. Consequences of maternal morbidity on health-related functioning: a systematic scoping review. BMJ Open 2017;7:e013903 http://bmjopen.bmj.com/ content/7/6/e013903.abstract

5 NICE. Antenatal care for uncomplicated pregnancies [Internet], 2019. Available: https://www.nice.org.uk/guidance/cg62

6 Brasil. Secretaria de Atenção Saúde. Departamento de Atenção Básica. Atenção ao pré-natal de baixo risco Ministério da Saúde 2012.

7 Department of Health R of SA. Guidelines for Maternity Care in South Africa 2007 : A manual for clinics, community health centres and district hospitals. [Internet], 2016. Available: https://www. knowledgehub.org.za/elibrary/guidelines-maternity-care-southafrica-2016

8 World Health Organization (WHO). Who recommendations on antenatal care for a positive pregnancy experience, 2016.

9 Bowling A. Measuring health: a review of subjective health, wellbeing and quality of life measurement scales. 4 th. Maidenhead, Berkshire, United Kingdom.: Open University Press.

10 Ware JEJ, Sherbourne CD. The mos 36-item short-form health survey (SF-36). I. Conceptual framework and item selection. Med Care 1992;30:473-83.

11 WHO. Introduction, administration, scoring and generic version of the Assessment Field Trial Version December 1996 Programme on Mental Health. 1996;(December). Available: https://www.who.int/ mental health/media/en/76.pdf

12 Üstün TB. Measuring Health and Disability: Manual for WHO Disability Assessment Schedule WHODAS 2.0. World Heal Organ [Internet]. 2010;90. Available: https://books.google.com/books?hl= en\&l $\mathrm{r}=\& \mathrm{id}=\mathrm{h} 9 \mathrm{fhLNiaRTgC \& pgis=1}$

13 Embaby H, Elsayed E, Fawzy M. Insulin sensitivity and plasma glucose response to aerobic exercise in pregnant women at risk for gestational diabetes mellitus. Ethiop J Health Sci 2016;26:409-14.

14 Engberg E, Stach-Lempinen B, Rönö K, et al. A randomized lifestyle intervention preventing gestational diabetes: effects on self-rated health from pregnancy to postpartum. J Psychosom Obstet Gynaecol 2018;39:1-6.

15 Angelini CR, Pacagnella RC, Parpinelli MA, et al. Quality of life after an episode of severe maternal morbidity: evidence from a cohort study in Brazil. Biomed Res Int 2018;2018:9348647.

16 Türkmen $\mathrm{H}$. The effect of hyperemesis gravidarum on prenatal adaptation and quality of life: a prospective case-control study. $J$ Psychosom Obstet Gynaecol 2020;41:282-9.

17 Fazio AF. A concurrent validational study of the NCHS General wellbeing schedule. Vital Health Stat 2 1977;73:1-53.

18 Hill PD, Aldag JC, Hekel B, et al. Maternal postpartum quality of life questionnaire. J Nurs Meas 2006;14:205-20.

19 Ware J, Kosinski M, Keller SD. A 12-Item short-form health survey: construction of scales and preliminary tests of reliability and validity. Med Care 1996;34:220-33.

20 Fawcett J, Tulman L, Myers ST. Development of the inventory of functional status after childbirth. J Nurse Midwifery 1988;33:252-60.

21 Alderdice F, Savage-McGlynn E, Martin C, et al. The prenatal distress questionnaire: an investigation of factor structure in a high risk population. J Reprod Infant Psychol 2013;31:456-64.
22 Cox JL, Holden JM, Sagovsky R. Detection of postnatal depression. development of the 10-item Edinburgh postnatal depression scale. Br J Psychiatry 1987;150:782-6.

23 Lacasse A, Bérard A. Validation of the nausea and vomiting of pregnancy specific health related quality of life questionnaire. Health Qual Life Outcomes 2008;6:32.

24 Thomas JC, Silvestre E, Salentine S, et al. What systems are essential to achieving the sustainable development goals and what will it take to marshal them? Health Policy Plan 2016;31:1445-7.

25 Liberati A, Altman DG, Tetzlaff J, et al. The PRISMA statement for reporting systematic reviews and meta-analyses of studies that evaluate healthcare interventions: explanation and elaboration. BMJ 2009;339:b2700 https://www.bmj.com/content/339/bmj.b2700

26 Downe S, Finlayson K, Tunçalp Ö, et al. What matters to women: a systematic scoping review to identify the processes and outcomes of antenatal care provision that are important to healthy pregnant women. BJOG 2016;123:529-39.

27 GRADE. The GRADE Working Group [Internet]. Available: https:// www.gradeworkinggroup.org/ [Accessed cited 2020 Dec 1].

28 GRADE-CERQual. The GRADECERQual Project Group [Internet] Available: https://www.cerqual.org/the-grade-cerqual-project-group/ [Accessed cited 2020 Dec 1].

29 Sterne JAC, Savović J, Page MJ, et al. Rob 2: a revised tool for assessing risk of bias in randomised trials. BMJ 2019;366:14898 https://www.bmj.com/content/366/bmj.14898

30 Barreix M, Barbour K, McCaw-Binns A, et al. Standardizing the measurement of maternal morbidity: pilot study results. Int $J$ Gynaecol Obstet 2018;141 Suppl 1:10-19.

31 Muggah E, Graves E, Bennett C, et al. Ascertainment of chronic diseases using population health data: a comparison of health administrative data and patient self-report. BMC Public Health 2013;13:16.

32 Jiang L, Zhang B, Smith ML, et al. Concordance between SelfReports and Medicare claims among participants in a national study of chronic disease self-management program. Front. Public Health 2015;3:222

33 Mogos MF, August EM, Salinas-Miranda AA, et al. A systematic review of quality of life measures in pregnant and postpartum mothers. App/ Res Qual Life 2013;8:219-50 https://pubmed.ncbi. nlm.nih.gov/23734167

34 Langer A, Meleis A, Knaul FM, et al. Women and health: the key for sustainable development. Lancet 2015;386:1165-210.

35 Silveira C, Parpinelli MA, Pacagnella RC, et al. A cohort study of functioning and disability among women after severe maternal morbidity. Int J Gynaecol Obstet 2016;134:87-92.

36 Angelini CR, Pacagnella RC, Parpinelli MA, et al. Post-Traumatic stress disorder and severe maternal morbidity: is there an association? Clinics 2018;73:e309.

37 Adanu RMK. The obstetrician-gynecologist and the sustainable development goals. Int J Gynecol Obstet 2018;140:1-2.

38 Health. R of SAD of. Guidelines for maternity care in South Africa, 2015.

39 ACOG - American College of Obstetricians and Gynecologists. Guidelines for Perinatal Care [Internet], 2017. Available: https://www. healthplan.org/application/files/4415/6702/0206/GuidelinesforPerinat alCare

40 FIGO - The International Federation of Gynecology and Obstetrics. Committee for safe motherhood and newborn health, 2019.

41 SOGC - The Society of Obstetricians and Gynecologists of Canada. Clinical Practice Guidelines And Position Statements during Pregancy [Internet], 2019. Available: https://www.sogc.org/en/ content/guidelines-jogc/guidelines-and-jogc.aspx?hkey=aa09f7537812-462a-9d80-3e6b609f6ec6

42 Canberra - Australian Government Department of Health. Department of health (2018. Clinical Practice Guidelines: Pregnancy Care, 2019

43 CNGOF - Collège National des Gynécologues et Obstétriciens Français. Clinique guidelines, 2019.

44 Metelko Z, Szabo S, Diseases M, et al. The world Health organization quality of life assessment (WHOQOL). Position paper from the World Health Organization. Soc Sci Med 1995;41:1403-9. 